\title{
What Is the Tachycardia Mechanism?
}

\author{
FRED MORADY, M.D.
}

From the Division of Cardiology, The University of Michigan Medical Center, Ann Arbor, Michigan

\section{Case Presentation}

A 40-year-old man underwent an electrophysiologic procedure because of recurrent paroxysmal supraventricular tachycardia. Tachycardia with a cycle length of $290 \mathrm{msec}$ was induced by atrial overdrive pacing at a cycle length of $300 \mathrm{msec}$. The tachycardia was reproducibly terminated by a ventricular extrastimulus introduced at a coupling interval of 240 msec (Fig. 1). What is the mechanism of this tachycardia?

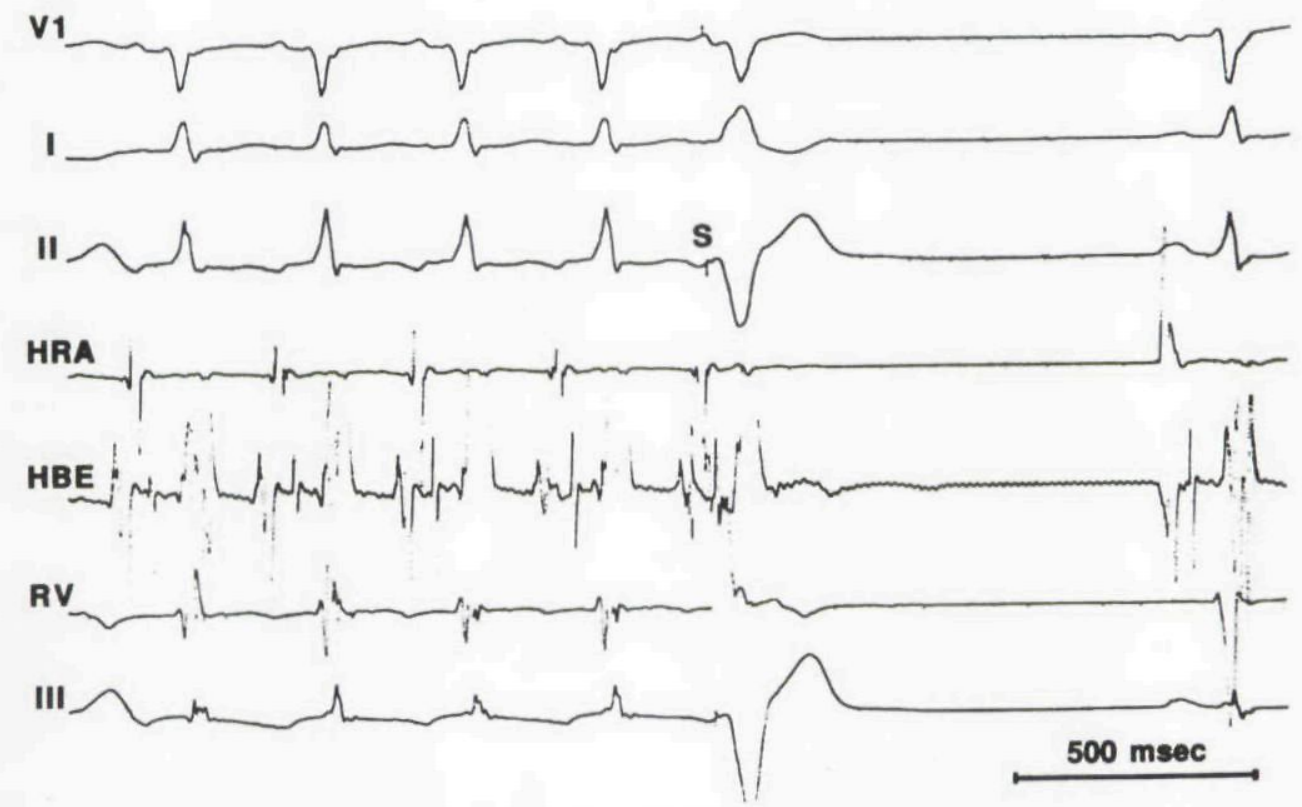

Figure 1. A narrow QRS tachycardia is terminated by a ventricular extrastimulus introduced at the right ventricular apex. Shown are leads VI, I, and II, high right atrial electrogram (HRA), His-bundle electrogram (HBE), right ventricular electro$\operatorname{gram}(R V)$, and lead III.

J Cardiovasc Electrophysiol, Vol. 6, pp. 863-864, October 1995, Pt. I.

Address for correspondence: Fred Morady, M.D., Division of Cardiology BL F245, The University of Michigan Medical Center,
1500 E. Medical Center Drive, Ann Arbor, MI 48109-0022. Fax: 313-936-7641.

Manuscript received 3 August 1995; Accepted for publication 4 August 1995. 


\section{Commentary}

This is a long RP tachycardia that has inverted $\mathrm{P}$ waves in the inferior leads. The differential diagnosis includes atrial tachycardia, atypical ("short-long") AV nodal reentrant tachycardia, and orthodromic tachycardia utilizing a relatively slowly conducting accessory pathway. Note that the tachycardia was terminated without premature depolarization of the atrium. The fact that the tachycardia was terminated by a ventricular extrastimulus that did not result in premature depolarization of the atrium excludes the possibility of an atrial tachycardia. Also note that the ventricular extrastimulus that terminated the tachycardia was simultaneous with the His-bundle electrogram. Because the reentrant circuit of AV nodal reentrant tachycardia occurs above the level of the His bundle, a ventricular depolarization that occurs when the His bundle is refractory would not be able to penetrate the reentrant circuit and terminate the tachycardia. The ability to terminate a narrow QRS tachycardia when the His bundle is refractory indicates that an extranodal pathway is present. Therefore, the response to the ventricular extrastimulus strongly suggests that the tachycardia is orthodromic.

Is any other tachycardia mechanism possible? A much less likely possibility would be atypical AV nodal reentrant tachycardia with a nodoventricular pathway that is an "innocent bystander" and that might allow a ventricular depolarization to have access to the AV node even when the His bundle is refractory. However, an innocent bystander nodoventricular pathway would be expected to undergo at least partial anterograde penetration during AV nodal reentrant tachycardia, making it unlikely that the pathway would be available for retrograde conduction when the His bundle was refractory. The remote possibility of AV nodal reentrant tachycardia with an innocent bystander nodoventricular pathway would be eliminated if an eccentric pattern of atrial activation were found during the tachycardia.

Activation mapping demonstrated that the earliest atrial activation during tachycardia was at the posterolateral aspect of the mitral annulus, consistent with retrograde conduction through a left posterolateral accessory pathway. The tachycardia was no longer inducible after radiofrequency catheter ablation of the accessory pathway. 
This document is a scanned copy of a printed document. No warranty is given about the accuracy of the copy. Users should refer to the original published version of the material. 